\title{
Determining the optimal periodicity for preventive replacement of mechanical spare parts
}

\author{
R. Berrehal*, S. Benissaad** \\ *Mechanical laboratory, Mechanical Engineering Department, Faculty of Technology, Université des Frères \\ Mentouri-Constantine, Algeria, E-mail: berrehalryma@yahoo.fr \\ **Laboratory of Applied Energy and Pollution, Mechanical Engineering, Department, Faculty of Technology, \\ Université des Frères Mentouri-Constantine, Algeria, E-mail: benissaad.smail@gmail.com
}

cross $^{\text {ref }}$ http://dx.doi.org/10.5755/j01.mech.22.2.12269

\section{Nomenclature}

$C_{r}$ - cost ratio of system preventive replacement to cost of system failure; $C_{f}$ - $c$ ost of system failure, DA; $C_{p}$ - cost of system preventive replacement, DA; $C(T)$ - total cost per unit time, DA/h; CM - corrective maintenance; $f(t)$ - failure density of the system, $f(t)=R^{\prime}(t) ; F(t)$ - unreliability of the system over time $t ; k$ - number of failure in one preventive replacement; $R(t)$ - reliability of the system over time $t$, $F(t)=1-R(t) ; M(T)$ - renewal function of block replacement policy; MTBF-mean time between failure; PM - preventive maintenance; $t$ - time, h; $T$-replacement time, $\mathrm{h} ; T_{0}$ - periodicity optimal of replacement, $\mathrm{h}$; $\beta$-weibull distribution shape parameter; $\varepsilon$-fraction of time, $\varepsilon<<T_{0}$, h; $\gamma$ - weibull distribution location parameter, $\mathrm{h} ; \eta$ - weibull distribution scale parameter, $\mathrm{h} ; \mu$-variable, $\mu=(t / \eta)^{\beta} ; y(\mu)$ - function of cost ratio; $\mu_{0}, \mu_{1}$ - roots of function $y(\mu)$.

\section{Introduction}

It is of great importance to avoid the failure of a system during actual operation when such an event is costly and/or dangerous. Almost all systems deteriorate with age and usage and exhibit stochastic failures during operation. Deterioration causes higher operating costs and produces less competitive goods. Further, because consecutive failures are dangerous to the system, timely preventive maintenance is necessary to support normal and continuous system operation. For these reasons, an important area of reliability theory is the study of various maintenance policies that seek the way to reduce operating costs and the risk of a catastrophic breakdown. So, Al-Najjar [1] showed that maintenance expenses vary depending on the type of industry; figures typically encountered are in the order of $15-40 \%$ of production costs. Therefore, it is necessary to pay more attention to this important subject area. Furthermore, timely preventive maintenance (or replacement) is also beneficial to support normal and continuous system operation. Therefore, it becomes desirable to determine an optimal replacement policy for the system.

Determining the optimal periodicity for preventive replacement of mechanical spare parts, components, or other systems always causes a problem in both economy and production. This economic problem is favored whenever the equipment is similar or the machines are identical. The so- lution of this problem resolved in the knowledge of the operational reliability and the determination of the most appropriate time to accomplish this preventive replacement. For this reason, different models have been proposed in the area of planning preventive preservation in order to find out optimal replacement policies.

An age-replacement policy, where an operating unit is replaced at time of failure, or at age $t$, whichever comes first, was proposed by Barlow and Proschan [2]. Another well-known preventive replacement policy is the periodic replacement policy, where an operating unit is replaced with a new one at periodic time or at failures. After these authors [2], systemically developed the age replacement policy, various situations have been intensely investigated for policy variations and extensions, and many analytical results have been obtained. Usually, in preventive maintenance planning the goal is to improve some important criteria of performance evaluation such as reparation costs, reliability and availability.

Recently, Yeh et al. [3] have analyzed the effects of the renewable free-replacement warranty (RFRW) on the age replacement policy, applicable to non-repairable products with increasing failure rates. The RFRW policy is one of the most common types of warranty policies, which is usually applied to non-repairable products. Under the RFRW policy, a product that fails within the warranty period is replaced, comes with a full warranty, and is free of charge to the buyer. They have developed cost models for both warranted and non-warranted products, and have derived corresponding optimal replacement ages, as based on minimized long-run expected cost rates. Chien [4] has also proposed and analyzed a new warranty strategy, which combines a renewable free-replacement warranty with a rebate policy. In this paper, determination of the optimal preventive replacement age $t$ for non-repairable products under a fully renewable FRW/PRW policy is developed. Denote $w$ as the warranty length of the product. Under the fully renewable FRW/PRW policy, the seller agrees to replace a product that fails prior to time point $w_{0}$, where $w_{0}>w$, from the time of purchase with a new product at no cost to the buyer. Meanwhile, any failure in the time interval from $w_{0}$ to $w$ results in a pro-rata replacement. In other words, any product is replaced with a new item at pro-rata cost to the buyer separate pre-specified age for preventive replacement beyond the warranty, during the PRW period or within the FRW period cost models from the user/buyer perspective are developed, and the corresponding local optimal replacement ages 
are derived such that long run expected cost rates are minimized.

Nosoohi and Hejazi [5] have presented a novel multi-objective model for preventive replacement of a part over a planning horizon. The proposed model considers different objectives and practical issues, such as corrective replacement and its consequences, residual lifetime objective, and somehow productivity index. Also, the model determines a number of spare parts, required for replacement with the defected part, to be provided at the beginning of the planning horizon. The multi-objective model is applicable for machines or equipments which are repaired through replacing their defected part with new spare part.

Halim and Tang [6] have extensively studied the replacement problems of deteriorating systems. Typically, the time between failures is characterized by lifetime distribution in which parameters are estimated from historical data. On the other hand, in most cases, the work focuses on determining the optimal replacement schedule by assuming that model parameters are constant. Here, the issues arising from the use of estimated parameters are studied and the results are applied to opportunistic replacement.

Jung and Park [7] have developed the optimal periodic preventive maintenance policies following the expiration of warranty. They have considered two types of warranty policies to discuss such optimum maintenance policies: renewing warranty and non-renewing warranty. From the user's perspective, the product is maintained free of charge or with prorated cost on failure during the warranty period. However, the users will have to repair or replace the failed product at their own expenses during the post-warranty period. Given the cost structure to the user during the cycle of the product, they derive the expressions for the expected maintenance costs for the periodic preventive maintenance following the expiration of warranty when applying two types of warranty policies and obtain the optimal number and the optimal period for such post-warranty maintenance policies by minimizing the expected long-run maintenance cost per unit time.

Chien and Chen [8] have presented a spare ordering policy for preventive replacement with age-dependent minimal repair and salvage value consideration. The spare unit for replacement is available only by order and the leadtime for delivering the spare due to regular or expedited ordering follows general distributions. To analyze the ordering policy, the failure process is modeled by a non-homogeneous Poisson process. By introducing the costs due to ordering, repairs, replacements and downtime, as well as the salvage value of a non-failed system, the expected cost of effectiveness in the long run are derived as a criterion of optimality.

Barlow and Hunter [9] have proposed two mathematical models for the determination of the policy of optimal replacement minimizing the cost operation of the production system. These models are called Block Replacement Models and Age Replacement Models

For block replacement models, the preventive replacement is executed periodically at a prespecified time $k T_{0}\left(T_{0} \geq 0\right)$ or $k N(N=0,1,2, \ldots),(k=1,2,3, \ldots)$. If the unit fails during the time interval $\left.\left((k-1) T_{0}, k T_{0}\right)\right]$ or $((k-1) N, k N)]$, then the corrective maintenance is made at the failure time. The main property for the block replacement is that it is easier to administer in general, since the preventive replacement time is programmed to the in advanced and we do not need the watch of the system age. There are three variations of block replacement model: a failed unit is replaced instantaneously at failure (type I); a failed unit remains inoperable until the next scheduled replacement comes (type II) or a failed unit undergoes minimal repair (type III).

In the age replacement model, as it is well recognized, if the unit does not fail until a prespecified time $T_{0}\left(T_{0} \geq 0\right)$ or $N(N=0,1,2, \ldots)$, then it is replaced by a new one preventively; otherwise, it is replaced at the failure time. This model plays a central role in all replacement models, since the optimality of the age replacement model has been proved by Bergman [10] if the replacement by a new unit is the only maintenance option (i.e. if no repair is considered as an alternative option).

This work aims at studying and determining the most appropriate period, from an economic point of view, to make replacements of mechanical parts. We take in consideration all the parameters that involved, so that, this operation can be profitable. We propose an analytical and numerical method for solving the resulting differential equation and we give some numerical examples.

\section{Mathematical model}

The aim of this study is to determine the periodicity $T_{0}$ with we should execute a preventive maintenance to minimize the operation cost.

We consider a type of parts on $n$ systems, we note as $C_{P}$ the cost of operation and $C_{f}$ the cost of replacement due for failure of part at time $T_{0}$.

This case is given by the following expression:

$$
C(T)=\frac{C_{p}+C_{f} M\left(T_{0}\right)}{T_{0}} ; T_{0} \geq 0,
$$

where the function $M\left(T_{0}\right)$ denotes the mean number of failures during the time period $(0, T]$ :

$$
M\left(T_{0}\right)=\sum_{k=1}^{\infty} F^{k}(T)
$$

and $F_{k}(T)$ the $k$-fold convolution of the lifetime distribution. We have:

$$
M\left(T_{0}\right)=F(T) .
$$

So let's think this case, proceed as follows:

- all parts are changed with the same periodicity $T_{0}$ whatever the age of the piece (Fig. 1), where $S_{1}, R, f$, and $T_{0}$ denote the system number 1 , a replacement, a failure and the replacement periodicity, respectively;

- the limiting case being that of a spare part changed at $T_{0}-\varepsilon$ for failure to and replaced at $T_{0}$ for preventive maintenance $\left(\varepsilon<<T_{0}\right)$;

- the operation cost will $C_{p}$ plus the eventual replacement cost due to a failure of the item during $T_{0}$, that is: 
$C_{f} P\left(T \leq T_{0}\right)=C_{f} F\left(T_{0}\right)$.

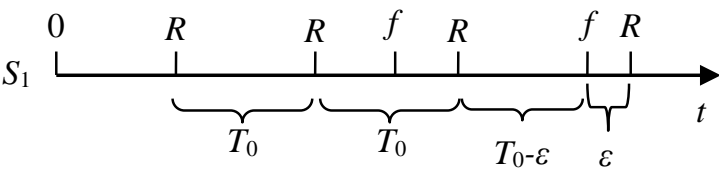

Fig. 1 Fixed period $T_{0}$ replacement

The total cost per part is:

$$
C(T)=C_{P}+C_{f}\left[1-R\left(T_{0}\right)\right] .
$$

This is an optimistic calculation because we considered only one failure during the period of time. It is the case more running in practice.

The average total costs by part and unit of time will be:

$$
C(T)=\frac{C_{p}+C_{f}\left[1-R\left(T_{0}\right)\right]}{T_{0}} .
$$

The problem is, of course, to derive the optimal block replacement time $T_{0}$ that minimizes $C(T)$.

$$
\begin{gathered}
C_{m}(T)=C^{\prime}(T)=\frac{T f(t) C_{f}-\left[C_{p}+C_{f}\left(1-R\left(T_{0}\right)\right)\right]}{t^{2}} ; \\
C_{m}(T)=0 \Leftrightarrow C_{f}\left[T f(t)+R\left(T_{0}\right)\right]=C_{p}+C_{f} \\
T f(T)+R\left(T_{0}\right)=1+\frac{C_{p}}{C_{f}}=1+C_{r} .
\end{gathered}
$$

It is well known that the law reliability that adjusts best parts and mechanical equipment is the Weibull distribution. It is best adapted to adjust the wear and old age phenomena. It is a law to three parameters: $\gamma, \beta$ and $\eta$ which aptly describe the behavior of the material studied. In addition it's a general law that includes the exponential law with a single parameter $\lambda$. In the case where $\gamma=0$ and $\beta=1$, we have $\lambda=1 / \eta$. That is why we have chosen the use of Weibull distribution in this study.

Then, in the case of Weibull law with $\gamma=0$.This means that the origin of time is taken equal at zero, and the equipment was operated at $t=0$. It's the most common case in practice. So we have:

$$
R(T)=e^{-\left(\frac{t}{\eta}\right)^{\beta}}
$$

and Eq. (9) becomes:

$$
t \frac{\beta}{\eta}\left(\frac{t}{\eta}\right)^{\beta-1} e^{-\left(\frac{t}{\eta}\right)^{\beta}}+e^{-\left(\frac{t}{\eta}\right)^{\beta}}=1+C_{r}
$$

or:

$$
\beta\left(\frac{t}{\eta}\right)^{\beta} e^{-\left(\frac{t}{\eta}\right)^{\beta}}+e^{-\left(\frac{t}{\eta}\right)^{\beta}}=1+C_{r} .
$$

We put:

$$
\left(\frac{t}{\eta}\right)^{\beta}=\mu \text {. }
$$

We obtained:

$e^{-\mu}(\beta \mu+1)-1=C_{r}$.

We put:

$$
y(\mu)=e^{-\mu}(\beta \mu+1)-1=C_{r} .
$$

\section{Analytical solution}

The analytical study of this equation shows:

- for $0<\beta<1$ : this case has no practical interest, since the material is in youth period;

- for $\beta=1$ : this case of an exponential law $\lambda=1 / \eta$ , it is a fatigue material or during operation. The only solution to the Eq. (15) corresponds to $C_{p}=0$, which has no practical interest;

- for $\beta>1$ : the equation has two solutions:

$$
\begin{aligned}
& t_{0}=\eta \mu_{0}^{\frac{1}{\beta}} ; \\
& t_{1}=\eta \mu_{1}^{\frac{1}{\beta}} .
\end{aligned}
$$

With condition:

$$
0<C_{r}<\beta e^{-\left(\frac{\beta-1}{\beta}\right)}-1 .
$$

According to the Eq. (7):

$$
C_{m}(T)=C_{f} \frac{\left(t f(t)+R(t)-1-C_{r}\right)}{t^{2}} .
$$

From the expression for $y(\mu)$ was (Eqs. 13 and 15):

$$
C_{m}(T)=C_{f} \frac{\left(y(\mu)-C_{r}\right)}{t^{2}} .
$$

It is then observed for (Fig. 2):

$$
\begin{aligned}
& 0<\mu<\mu_{0}: C_{m}(T)<0 ; \\
& \mu_{0}<\mu<\mu_{1} ; y(\mu)>C_{r}: C_{m}(T)>0 ; \\
& \mu>\mu_{1} ; y(\mu)<C_{r}: C_{m}(T)<0 .
\end{aligned}
$$

We obtain the following graph of $C(T)$ (Fig. 3). The solution will be:

$$
t_{0}=\eta \quad \mu_{0}^{1 / \beta} .
$$




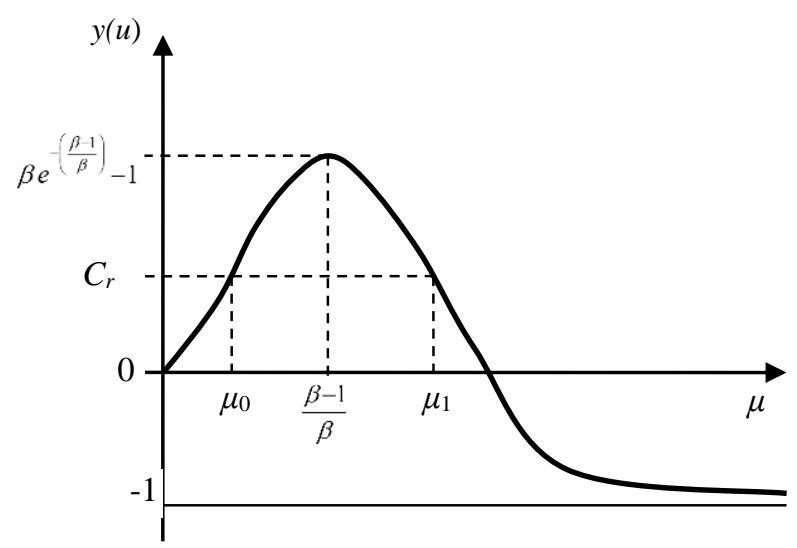

Fig. 2 Curve of $y(\mu)$ in the case $\beta>1$

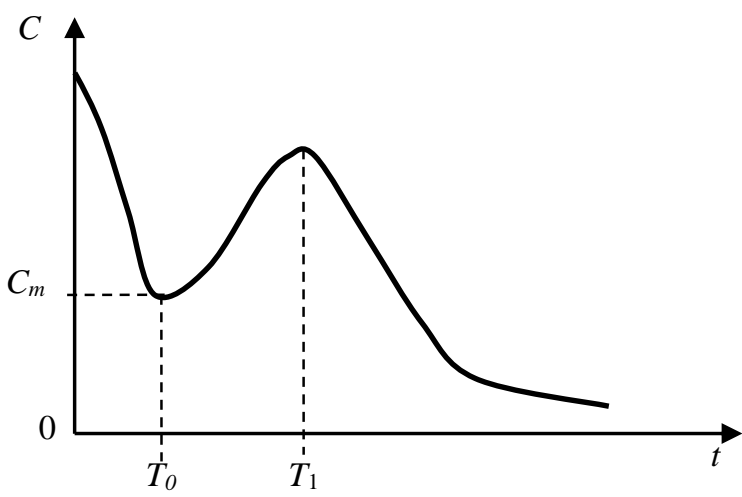

Fig. 3 Curve of in $C_{m}(t)$ the case of $\beta>1$

\section{Numerical solution}

To find $\mu_{0}$ and $\mu_{1}$ roots of the Eq. (15) and derivate the optimal periodicity of replacement $T_{0}$ we have to solve directly the Eq. (18) and obtain $T_{0}$ and $T_{1}$ roots or solve the Eq. (15) and obtained $\mu_{0}$ and $\mu_{1}$ from which we deduce $T_{0}$ and $T_{1}$, because the analytical solution of these equation is difficult. For this, we use numerical methods to find the roots of these differential equations and obtain the value of $T_{0}$.

In this work we have chosen to use a direct method to obtain the roots of Eq. (18) and find $T_{0}$ for define the minimum cost while meeting the condition (17).

An iterative procedure is employed to solve the Eq. 18). Our numerical code of calculation was validated by comparison with our analytical analysis and with the numerical results available in literature [11].

\subsection{Effect of the shape parameter}

We present, then, the results obtained by the numerical resolution of the differential equation which expressed the average total cost per unit of time and by spare part and which gave place to the Eq. (18) in the case of Weibull law. These results relate to the effect of the cost ratio of system preventive replacement to cost of system failure, $\mathrm{C}$, according the form parameter, $\beta$, on the value of the periodicity of optimal replacement $T_{0}$. Thus in each situation, we vary these parameters, while the other parameters will be maintained fixed.
We note, in Fig. 4, the presence of a minimal cost $C_{m}$ corresponding to the period most adapted to carry out the operation of PM. This cost, appreciably, depend on the value of the cost ratio and the form parameter $\beta$. It varies from 2.28 for $\beta=7$ to 4.09 for $\beta=2.5$ and the period of optimal replacement correspondent varies between 1110 for $\beta=2.5$ to 1280 for $\beta=7$.



Fig. 4 Variation of $C$ according to time for various values of $\beta: \eta=2000, C_{r}=0.25$

In this case, that the operations of PM are very beneficial as the values of $\beta$ are raised, this actually corresponds to a failure rate strongly growing (period of wear and old age). This period $T_{0}$, corresponding to the minimal cost, can be slightly differed, in the event of need. But, in the event of an excessive delay, the cost of the operation increases quickly.

Fig. 5 shows the variation of the mean total cost per unit time and per spare part over time in the case of $C_{r}=0.50$ for different values of $\beta$.

We note here that we do not have an optimal solution for $\beta<3$. For $\beta=3$, we remark that the period of PM starts functioning beyond $T=1535$ where $C$ is minimal (equal to 5.62) and it will constitute to be it as from this moment. While, for the other values of $\beta$, the value of the minimal cost $C_{m}$ corresponding to the period of PM varies from 4.13 for $\beta=7$ to 5.35 for $\beta=3.5$, and the period of optimal replacement correspondent varies between 1395 for $\beta=3.5$ to 1422 for $\beta=7$.

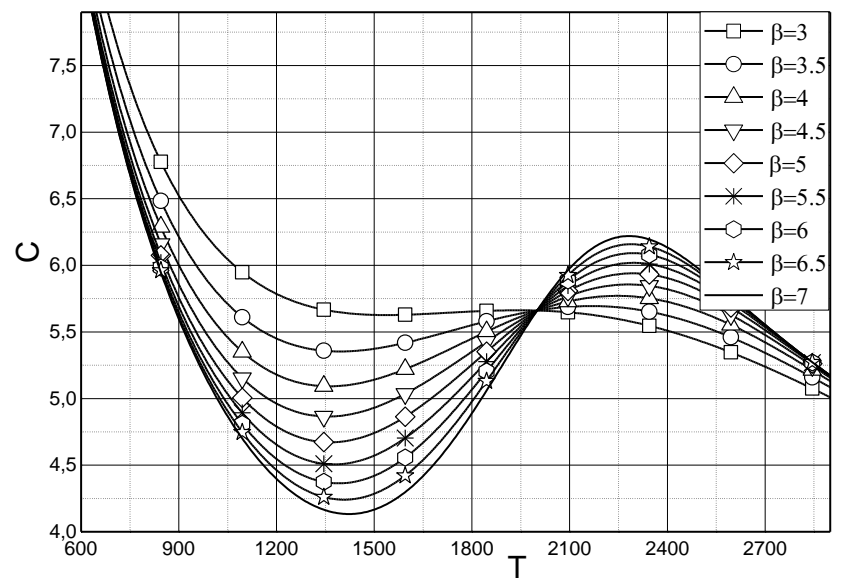

Fig. 5 Variation of $C$ according to time for various values of $\beta: \eta=2000, C_{r}=0.50$ 
Fig. 6 shows the variation of the average total costs per unit of time and part according to time in the case of $C_{r}=0.75$ for various values of $\beta$.

From this figure (Fig. 6), we can retain the same ones noted as the preceding case (Fig. 5). We note here that we do not have an optimal solution for $\beta<4$. We notice, that for $\beta=3$, the period of PM starts operating from $T=1600$ where $C$ is minimal (equal to 6.78) and it will constitute to be it as from this moment until $T=1800$, where it will start to grow before decreasing at $T=2300$. When with the variation of $C$ according to the period replacement, we note that the increase in generates a weak variation of $C$. In this case where $C_{r}=0.75$, it is less important than in the two preceding cases $\left(C_{r}=0.25 ; 0.5\right)$.

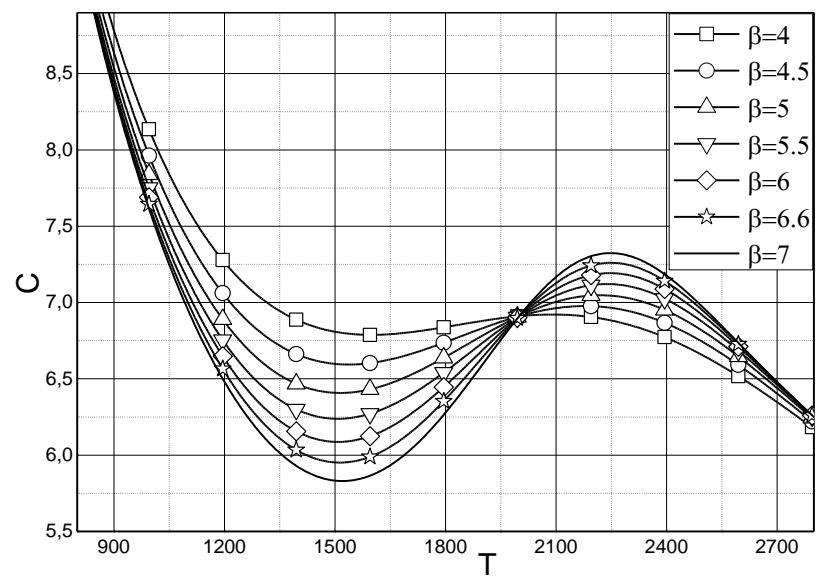

Fig. 6 Variation of $C$ according to time for various values of $\beta: \eta=2000, C_{r}=0.75$

The variation of the mean total cost per unit time $C$ according to time of $C=1$ and for various values of $\beta$ is presented on Fig. 7. This case is relatively similar to the precedent (case where $C=0.75$ ). We note here that we do not have an optimal solution for $\beta<4.5$. Whereas, for $\beta=4.5$, the period of PM starts functioning from $T=1745$ where $C$ is minimal and equal to 8.12 , there will remain constant until $T=2200$, value from which he starts to decrease. It is less important than in the three preceding cases. $C_{m}$ varies from 7.43 in the case of $\beta=7$ correspondent in $T=1600$, to 8.44 when $T=2215$.

When the cost of PM becomes higher than the

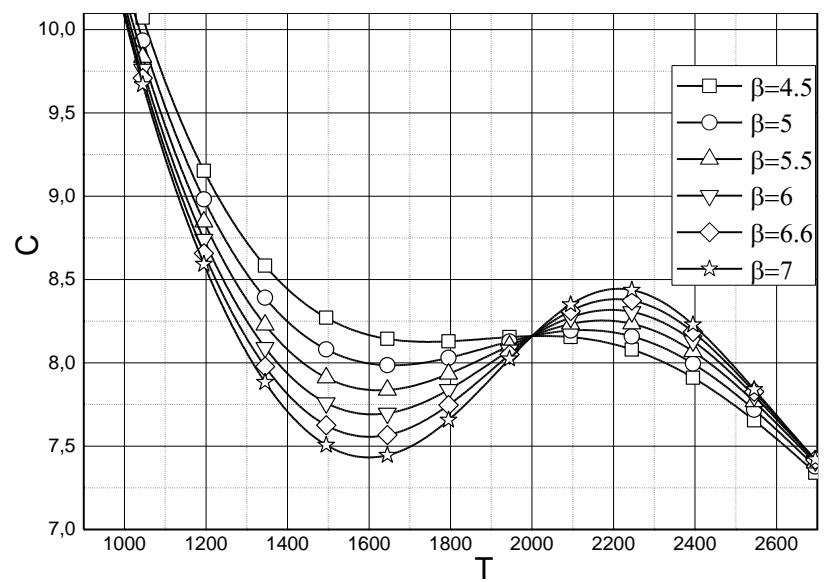

Fig. 7 Variation of $C$ according to time for various values of $\beta: \eta=2000, C_{r}=1$ cost of CM, the PM does not have any more economic interest. Fig. 8 shows the variation of the average total costs per time unit according to time in the case of $C_{r}=1.25$ for various values of $\beta$. We note according to this graph that $C$ does not have any more one minimum making it possible to determine $T_{0}$, some is the value of the parameter of form $\beta$. But, $C$ reaches its minimal value and will continue to decrease starting from a given value of $T$. This enables us to confirm, from an economic point of view, that the PM should not be programmed when its cost becomes higher than that of the CM.

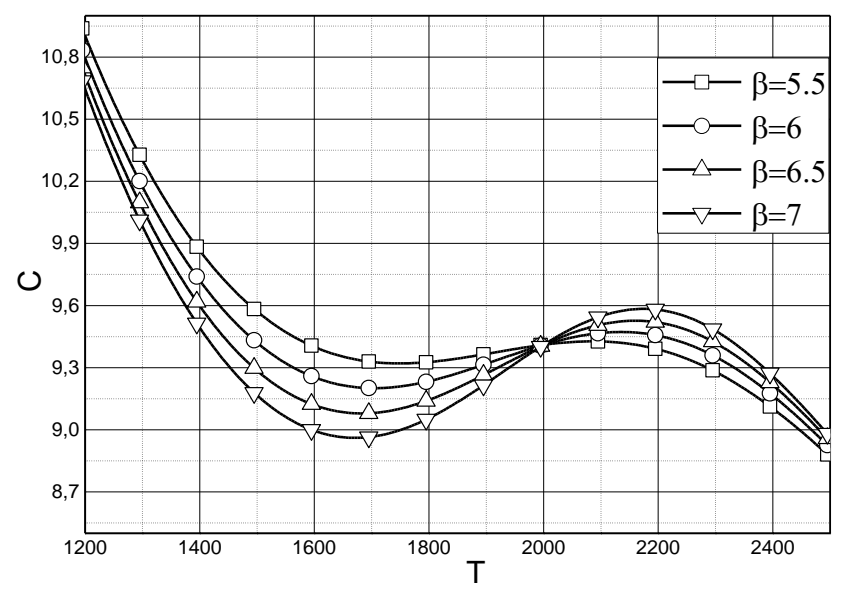

Fig. 8 Variation of $C$ according to time for various values of $\beta: \eta=2000, C_{r}=1.25$

\subsection{Effect of the scale parameter}

Fig. 9 shows the variation of the mean total cost per time unit $C$ according to time in the case of $C_{r}=0.5, \beta=5$ and for various values of the parameter of scale $\eta$. We note that $C$ decreases with the increase in the values of $\eta$, whereas the period optimal of replacement $T_{0}$ increases. Thus for $\eta=1500 ; C_{m}=6.2$ and $T_{0}=1000$, while for $\eta=5000$; $C_{m}=1.9$ and $T_{0}=3500$. Knowing that the parameter of scale represents an approximate value of MTBF, this evolution of $C$ is completely logical. Indeed, for a type of spare part having a MTBF relatively weak, its duration of exploitation is also small and $C_{m}$ is relatively high; whereas for a spare part which has a MTBF relatively high, its duration of exploitation is large and $C_{m}$ is minimal.

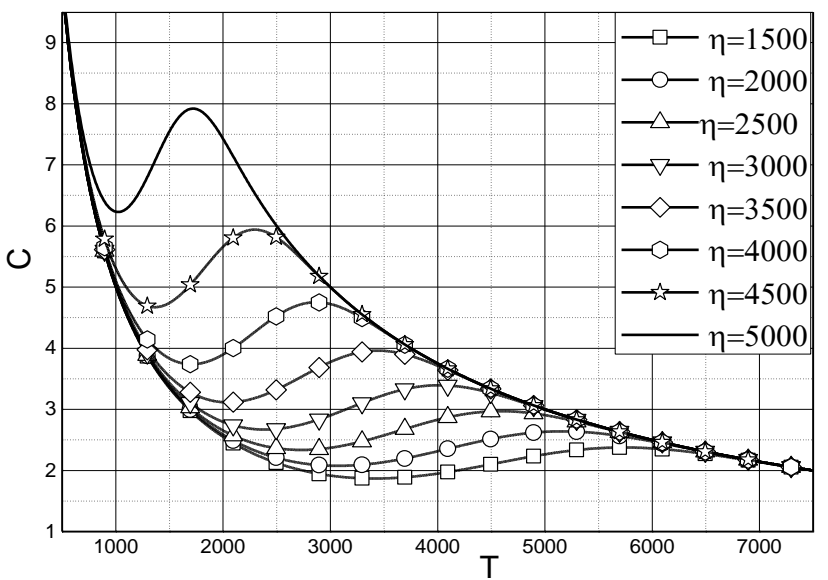

Fig. 9 Variation of $C$ according to time for various values of $\eta: \beta=5, C_{r}=0.5$ 


\section{Conclusion}

Determining the optimal periodicity for the preventive replacement might be obtained through two mathematical models: the replacement model by block and the replacement model based on age. Each model might lead to several variants. The standard is to calculate the average total cost per time unit and per item, to get the minimum period corresponding to this minimum as an optimal time to perform the preventive maintenance. This cost comprises the cost of preventive maintenance and the biased probability for the fault of corrective maintenance.

An analytical study that has been carried out in the case of a Weibull distribution and the resulting differential equation has been solved under certain mathematical conditions. After that, this equation has been numerically solved for the different parameters of this problem which are the cost ratio of maintenance, the scale parameter and the shape parameter.

The results were analyzed and discussed. Their applications to real cases can provide to maintenance service a key element in choosing the most suitable time to perform preventive maintenance at minimum cost.

\section{References}

1. Al-Najjar, B. 2003. Alsyouf, I.: Selecting the most efficient maintenance approach using fuzzy multiple criteria decision making, Int. J. Prod. Econ. 84(1): 85-100. http://dx.doi.org/10.1016/S0925-5273(02)00380-8.

2. Barlow, R.E.; Proschan, F. 1965. Mathematical theory of reliability, Wiley, New York, $256 \mathrm{p}$.

3. Yeh, R.H.; Chen, G.C.; Chen, M.Y. 2005. Optimal age-replacement policy for non-repairable products under renewing free-replacement warranty, IEEE Transactions on Reliability 54(1): 92-97. http://dx.doi.org/10.1109/TR.2004.841723.

4. Chien, Y.H. 2005. Determining optimal warranty periods from the seller's perspective and optimal out-ofwarranty replacement age from the buyer's perspective, International Journal of Systems Science 36(10): 361367.

http://dx.doi.org/10.1080/00207720500219054.

5. Nosoohi, I.; Hejazi, S.R. 2011. A multi-objective approach to simultaneous determination of spare part numbers and preventive replacement times, Appl. Math. Model. 3: 1157-1166. http://dx.doi.org/10.1016/j.apm.2010.08.003.

6. Halim, T.; Tang, L.C. 2009. Confidence interval for optimal preventive maintenance interval and its applications in maintenance planning, Int. J. Adv. Manuf. Technol. 40: 203-213.

http://dx.doi.org/10.1007/s00170-007-1315-2.

7. Jung, G.M.; Park, D.H. 2003. Optimal maintenance policies during the post-warranty period, Reliab. Eng. Syst. Safe. 82: 173-185. http://dx.doi.org/10.1016/S0951-8320(03)00144-3.

8. Chien, Y.H.; Chen, J.A. 2010. Optimal spare ordering policy for preventive replacement under cost effectiveness criterion, Appl. Math. Model. 34: 716-724. http://dx.doi.org/10.1016/j.apm.2009.06.017.

9. Bergman, B. 1980. On the optimality of stationary replacement strategies, J. App. Prob. 17: 78-86. http://dx.doi.org/10.2307/3212935.

10. Manzini, R.; Regattieri, A.; Pham, H.; Ferrari, E. 2010. Maintenance for Industrial Systems, Springer 314-339.

\section{R. Berrehal, S. Benissaad}

\section{DETERMINING THE OPTIMAL PERIODICITY FOR PREVENTIVE REPLACEMENT OF MECHANICAL SPARE PARTS}

S u m m a r y

The goal of this work is determining the periodicity $T_{0}$ which the preventive maintenance will execute for minimizing the operation cost. This type of preventive change is very used in practice by industrial manufacturing. In this study, we choose the model of block replacement type1: A failed unit is replaced instantaneously at failure. The mathematical model used is based on the Weibull law with $\gamma=0$. The results obtained are discussed according to the values of the parameters of the Weibull law $\beta, \eta$ and of the cost of preventive maintenance $C_{p}$ and cost failure $C_{f}$, for which the minimal cost $C_{m}$ has been determined in each case.

Keywords: optimal periodicity, preventive maintenance, corrective maintenance, failure, ratio cost, Weibull law.

Received May 15, 2015

Accepted March 15, 2016 